\title{
Neige d'avril
}

\section{Is abelle Armitage}

"A quelle heure rentreras-tu?" Agathe contint son impatience, "Vers quatre heures, comme d'habitude." Deux fois par semaine elle prenait des leçons d'espagnol et Martin posait toujours la même question. I1 ne se rappelait jamais ce qu'elle faisait, ça ne l'intéressait pas. Elle avait de la chance quand elle rentrait à temps pour l'empêcher de boire. S'il avait commencé trop tôt il était impossible d'éviter une scène à 1 'heure du dîner. Il refusait d'admettre qu'il avait un problème, et se moquait d'elle parce qu'elle ne tenait pas I'alcool.

Il avait sorti la voiture du garage laissant le moteur en marche. II ne le faisait pas vraiment pour l'aider, en fait elle aurait souvent préféré qu'il laissât la voiture à couvert jusqu'à ce qu'elle soit prête à partir. Mais elle était femme, donc elle ne connaissait rien aux mécaniques compliquées et ne comprenait pas qu'il faut chauffer un moteur, sinon $I^{\prime}$ huile n'est pas fluide et la fragile petite masse d'acier pourrait en souffrir d'une façon irréparable. Si seulement il avait eu autant de tendresse pour elle!

Le soleil d'avril était brillant, mais le fond de 1 'air était frais et elle mit son manteau ouatiné. Elle pensait au dicton de son enfance, "En avril ne te découvre pas d'un fil, en mai fait ce qu'il te plaît."

Agathe allait avoir quarante ans. Quand elle avait épousé Martin à New York, dix ans auparavant, elle n'avait jamais pensé qu'elle vivrait à Santa Fé. Elle travaillait dans la couture, et il lui avait fait croire qu'elle pourrait se faire une situation dans une revue de mode. Il était directeur artistique d'un magazine important; elle entrevoyait une association, une vie sophistiquēe, active. E1le s'était donné beaucoup de mal pour décorer leur appartement. Elle aimait la vieille maison de pierres brunes située en plein centre 
de Manhattan, avec son salon qui ouvrait sur un jardin. - Lais trois mois plus tard il était rentré du bureau et avait annoncé froidement qu'ils partaient pour le ranch. Il avait démissionné, "Je vieillis de cinq ans chaque année dans cette affreuse ville."

Il ne lui avait pas demandé son avis, ni ce qu'elle aimait. Elle était atterrée. E1le avait passé les années de guerre en France, dans la Résistance, vivant comme un animal pris au piège. Elle avait recommencé à vivre aux Etats-Unis et maintenant il voulait $I^{\prime}$ enterrer dans le désert de Californie, à vingt kilomètres du village le plus proche, sans téléphone, sans eau, sans électricité. Sans doute c'était un lieu spectaculaire, un hâvre où il était possible de se détendre après 1 'agitation de la grande ville, mais y vivre toute 1 'année!

Elle n'aimait pas déclarer forfait, en conséquence elle décida d'en prendre son parti. Elle refit une nouvelle installation. Elle devint excellente cuisinière car il y avait toujours de nombreux invités. Elle s'intéressa à toutes les entreprises de Martin, travaillant sans relâche à améliorer, à constuire, à entretenir, à lui apporter la joie et la sécurité. Elle arriva même à élever convenablement ses enfants.

Mais.elle ne pouvait pas gagner. Il vieillissait et il se faisait des illusions sur son activité, il ne savait pas quoi inventer pour s'occuper. Il la rendait responsable de ses échecs. Elle lui servait de bouc émissaire. D'ailleurs il la trouvait beaucoup trop organisée et artificielle. Ca l'étonnait qu'elle $\mathrm{n}$ 'aimât pas son costume de máison: caleçon, jottes et casquette de base-ball.

Un jour il était rentré malade d'un de ses rares voyages à Los Angeles et elle avait réussi à l'emmener à l'hôpital le plus proche, à soixante quinze kilomètres de là, pour le faire opérer d'urgence. Elle avait eu la paix pendant quelques temps après l'opération, et il avait finalement accepté le retour à la civilisation.

Tout en conduisant elle réfléchissait. La nouveauté du déménagement s'estompait. Leurs rapports empiraient, il n'avait que du mépris pour tout ce qu'elle essayait de faire. Les études, en particulier, lui semblaient du plus haut ridicule. Il riait des "petites gens" qui avaient besoin de diplômes. Il avait bien réussi, lui, 
et sans peau d'âne. Elle oublia ses problèmes grâce à l'effort qu'il lui fallait pour s'exprimer dans une langue qu'elle connaissait mal.

Deux heures plus tard quand elle sortit elle crut rêver. Sa voiture était couverte de neige. La tempête faisait rage et les flocons obscurcissaient le parebrise. Il devait y avoir dix centimètres de neige sur le sol et ses pieds étaient tout mouillés. Elle démarra et se dirigea à 1'aveuglette vers la maison. A la hauteur de la cathédrale elle eut le désir impulsif d'entrer prier. Elle n'était pas prête à confronter Martin. Elle voulait préserver quelques minutes de paix supplémentaires. Ce ne fut pas sans mal que la voiture accéda au sommet de la côte qui conduisait au parking derrière 1'église. Il était stupide de retarder ainsi son retour, mais elle n'était pas rationnelle, elle se moquait de 1'orage, elle voulait faire ce qui lui plaisait. E1le descendit de voiture et se précipita en courant vers la porte pour éviter d'être trempée. Elle glissa et tomba en arrière. Elle atterrit sur le coude gauche, le choc heureusement amorti par la doublure du manteau. Elle regarda autour d'elle, c'était une chance, personne ne $1^{\prime}$ avait vue étendue de tout son long dans la neige comme une idiote. Elle se releva toute raide, et elle tituba jusqu'à la vieille église.

Il lui fut impossible de prier, son bras la faisait trop souffrir. Elle se demanda si elle devrait aller directement à 1'hôpital, mais elle était blessée, elle ne voulait pas être seule. Elle désirait de toutes ses forces que Martin s'occupât d'elle. Elle crut qu'elle n'arriverait jamais jusqu'à la voiture. Il lui fallut dix minutes pour faire demi-tour dans la neige avec un seul bras et elle ramena la voiture au garage avec l'énergie du désespoir.

I1 était quatre heures et demie et Martin avait un verre à la main. Son regard bleu était dur et coléreux. Quoique corpulent il se tenait très droit et semblait grand bien qu'il ne mesurât qu'un mètre soixante dix. I1 lui fit penser à un juge aux enfers, Eaque peut-être. Son désir d'être protégée vola en éclats, elle était condamnée.

"Je suis tombée, mon bras me fait affreusement mal." "Tu es tombée? Sur le dos? Il faut que j'appelle le 
kinésithérapeute immédiatement. Ta colonne vertébrale doit être toute de travers." Martin détestait les docteurs en médecine, les pilules chimiques, il ne croyait qu'aux rebouteux, aux drogues naturelles. Elle le supplia, grelottant dans son manteau mouillé, elle sentait la nausée l'envahir. Il pourrait peut-être la conduire au service de secours à 1'hôpital. Ça ne coûterait rien puisqu'ils étaient assurés. Il était sourd. Sa colonne vertébrale . . le kinésithérapeute . . toute de travers. Les mots l'étourdissaient. Elle s'assit.

"Bon, appelle-le." "N'importe quoi," pensa-t-elle, "n'importe quoi, pour qu'il se taise."

"Oui, Docteur, je 1'amène tout de suite." "Viens," ordonna-t-i1, "un bon traitement et tu sera remise à neuf."

Ils s'assirent dans le salon d'attente surchauffé. Martin disparut derrière un numéro de Life. Elle ne pouvait ni lire, ni penser. Finalement on prit des radios, et comme Martin 1'avait prévu, son bras était en parfait état. On la fit monter et descendre des tables de traitement, tenant son bras "en parfait état" et sa colonne vertébrale fut craquée, tirée, tordue.

"Y a-t-il quelque chose que je puisse faire contre la douleur?" demanda-t-elle, sans espoir. "Faites des bains de sels d'epsom, ça s'arrangera."

Martin conduisait nerveusement et la voiture dérapait le long des rues boueuses et glacées. Il avait faim. Il attendit qu'elle soit couchée, sans d'ai1leurs I'aider à enlever la lourde couverture indienne qui lui servait de couvre-1it. I1 lui apporta les sels d'epsom que le docteur avait prescrits.

"Veux-tu quelque chose à manger?" Elle n'avait pas faim, mais même si ça n'avait pas été le cas la réponse qu'il attendait était "non".

Elle l'encouragea à sortir plutôt que d'avoir à nettoyer derrière lui s'il préparait son propre dîner.

En s'asseyant dans son lit elle constata que son côté gauche était mouillé. Elle souleva le bol en plastique dans lequel avait baigné son coude. Elle se rappela, c'était celui dans lequel Martin avait'fait la pâte à crèpes le dimanche précédent. I1 l'avait poussé trop près de la poële électrique et il y avait un petit 
trou sur la paroi. Son sens de l'humour prit le dessus et elle éclata de rire.

Elle se leva pour rapporter 1 'inutile objet dans 1 a la cuisine. Il lui était impossible de refaire son lit avec un seul bras et bien qu'elle essayât de se convaincre qu'il n'y avait rien de cassé chaque mouvement involontaire de son bras gauche la faisait hurler. Pourtant comme elle ne pouvait pas rester plantée là, elle retourna se coucher. Elle se rappela qu'elle devait inviter un certain nombre de dames par téléphone pour une réunion le dimanche suivant, et il y avait un appareil près de son lit. Cela 1'occupa jusqu'au retour de Martin. Il passa le nez dans la porte, ayant vérifié qu' elle était toujours là il avait satisfait son sens du devoir, et il se retira dignement dans ses appartements.

Elle avait encore un coup de téléphone à donner. Sa voix n'était pas très assurée et Marge s'en inquiéta. Elle lui raconta $1^{\prime}$ accident et son amie offrit de $1^{\prime} \mathrm{em}-$ mener à 1'hôpital. "Je vais demander à Martin et je te rappelle." Martin dormait déjà. Il avait bien bu et bien mangé. Elle pouvait aller sur la lune si ça lui plaisait. Le docteur Ramirez avait dit qu'elle n'avait rien. Si elle était hypocondre c'était son affaire.

Il fallut que Marge $I^{\prime}$ aide à s'habiller. Elle n'osait pas se regarder dans la glace, elle n'imaginait que trop bien ce à quoi elle ressemblait avec sa jupe rouge trop large, ses collants noirs et le vieux chandail dont sa fille avait presque totalement éliminé les coudes. Elle avait retiré son maquillage, et elle sentait les cernes noirs qui soulignaient ses yeux. Heureusement ses cheveux courts, faciles à coiffer, lui conservaient un semblant d'élégance. Marge lui jeta un manteau sur les épaules.

$\mathrm{Au}$ service de secours 1'infimière se moqua gentiment d'elle. Il était neuf heures et la chute s'était produite à quatre heures; un vrai cas d'urgence. Le processus se déroula, on appela le médecin de service qui prescrivit des radio, et on la mit dans un fauteuil roulant malgré ses protestations. A la sortie de l'ascenseur on la gara face au mur. Il fallait radiographier son bras de dos, de face et de profil, et chaque position était plus pénible que 1 'autre. On la roula de nouveau dans le corridor. Les radios furent dévelop- 
pées rapidement et le médecin crut détecter une fine fracture de la tête du radius.

"Il faut que vous appeliez un spécialiste, qui voulez-vous?" E1le donna le nom que lui souffla Marge. Vingt minutes plus tard une femme médecin au sourire rassurant fit son apparition. Elle prit la main gauche d'Agathe et la tordit légèrement. "Aie!" hurla Agathe. "Le médecin de service a raison, Vous avez une fracture, il faut que je mette un plâtre."

En quarante ans Agathe ne s'était jamais rien cassé, et le plâtre qualifié de léger lui semblait très lourd et plutôt malcommode, son bras était immobilisé à angle droit. La spécialiste prescrit un analgésique et lui donna rendez-vous pour la semaine suivante.

I1 était plus de onze heures quand Agathe rentra de 1'hôpital. L'affectueux pointer blanc se leva pour 1'accueillir et Martin le suivait, revêtu de sa veste de pyjama. "Marge est avec moi," le prévint-elle. Il fit une retraite hâtive, et attrappa sa robe de chambre bleue à pois blancs. Il rentra à grand bruit dans le salon. I1 portait ses bottes. "Il ne sera jamais adulte," pensa-t-elle.

"Vous voulez prendre un verre avec moi?" demandat-il à Marge. "Non merci, il est tard, il faut que je rentre." "J'en prendrais un volontiers," dit Agathe. I1 la regarda avec moquerie, "Tiens, tu te réformes?" I1 prit conscience de son plâtre et se livra à la plus grande hilarité." "Tu es contente, tu es infirme, si tu savais comme tu as I'air bête."

Marge était mal à son aise. Elle n'aimait pas la façon dont Martin la détaillait. Elle s'esquiva le plus poliment qu'elle put.

Ils étaient seuls. Agathe prit une longue gorgée de scotch et fit face à l'inévitable attaque. "Je t'avais prévenue, les docteurs en médecine sont des ânes. Qu'est-ce que tu vas faire avec un seul bras?" "Il est cassé, il fallait 1'immobiliser." "Sainte Agathe," persifla-t-il "pourquoi ton Dieu ne te guérit-il pas? Ne compte pas sur ma sympathie." C'était une sous-estimation de son attitude. Elle résista à l'envie de I'assommer avec son plâtre. Au moins il était franc. I1 avait tellement 1 'habitude de la maltraiter qu'il n'essayait même plus d'être aimable. Elle commençait à sen- 
tir les effets de 1'analgésique. "Bonsoir, je n'en feux plus."

Elle resta éveillée encore un moment. Les réverbères créaient des ombres chinoises dans l'énorme chambre. A plat sur le dos elle se sentait flotter vers le haut plafond. Les objets familiers étaient 1à. Les photos de ses enfants sur la longue table de nuit lui souriaient dans la demi-obscurité.

"Nous y voilà donc," constata-t-elle. "I1 se sent en sécurité, il pense que ma religion m'empêchera de me libérer. Mais qu'est-ce qu'il croit? Que d'être charitable signifie qu'il faut céder à l'injustice? Il a beaucoup à apprendre. Charité bien ordonnée commence par soi-même. Aime ton prochain comme toi-même. Je ferais aussi bien de commencer par moi." Et elle sombra dans un profond sommeil.

Quand elle se réveilla Martin était dans la pièce regardant son plâtre avec mépris. "Je veux divorcer," dit-elle calmement.

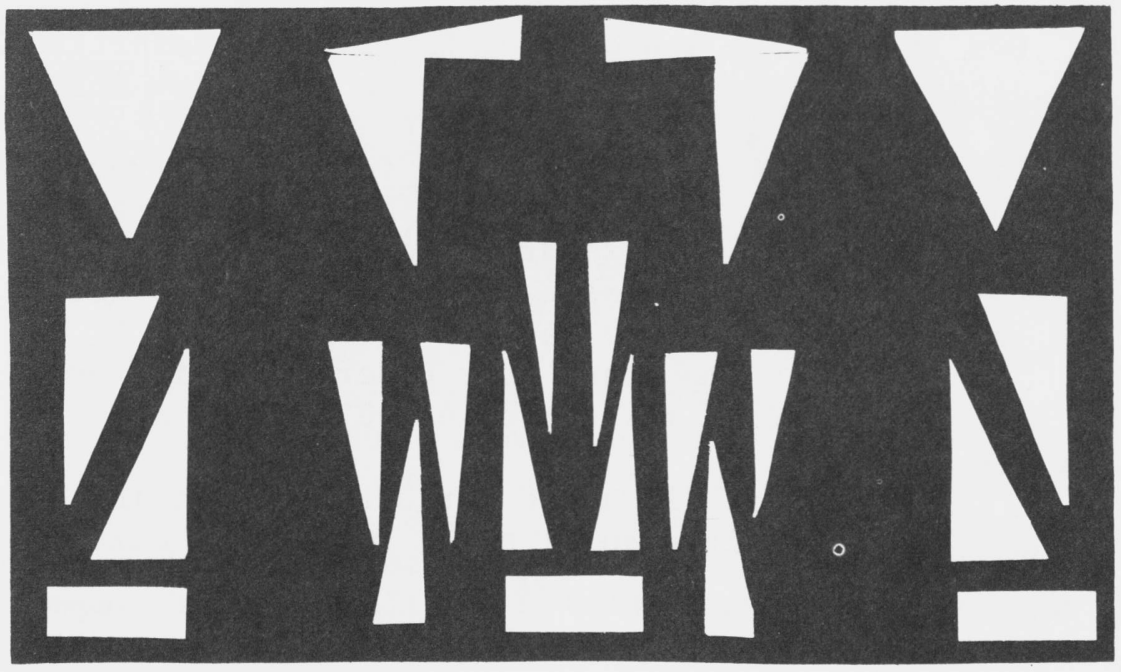

